\title{
Energy Harvesting Potential of Tuned Inertial Mass Electromagnetic Transducers
}

\author{
Takehiko Asai \\ Assistant Professor \\ Faculty of Engineering, Information and Systems \\ University of Tsukuba \\ Tsukuba, Ibaraki 305-8573, Japan \\ Email:asai@kz.tsukuba.ac.jp \\ Yoshikazu Araki \\ Associate Professor \\ Department of Architecture and Architectural Engineering \\ Kyoto University \\ Nishikyo, Kyoto 615-8540, Japan \\ Email: araki@archi.kyoto-u.ac.jp \\ Kohju Ikago \\ Professor \\ International Research Institute of Disaster Science \\ Tohoku University \\ Sendai, Miyagi 980-0845, Japan \\ Email: ikago@archi.tohoku.ac.jp
}

\begin{abstract}
The demand for developing renewable energy technologies has been growing in today's society. As one of promising renewable energy sources, large-scale energy harvesting from structural vibrations employing electromagnetic transducers has recently been proposed and considerable effort has been devoted to increase the power generation capability. In this paper, we introduce the mechanism of a tuned inertial mass electromagnetic transducer (TIMET), which can absorb vibratory energy more efficiently by tuning the parameters to adjust the system. Then we propose a new vibratory energy harvester with the TIMET and determine the parameter values for the device with a simple static admittance (SA) control law to maximize the energy harvested from a stationary stochastic disturbance. To investigate the energy harvesting potential of the TIMET further, the
\end{abstract}


performance-guaranteed (PG) control and the LQG control proposed in the literature are applied as well. Then the numerical simulation studies are carried out and the effectiveness of the proposed energy harvester is examined by comparing the traditional electromagnetic transducers.

Keywords: Energy harvesting, Tuned inertial mass electromagnetic transducer, Optimization

\section{Introduction}

Vibratory energy harvesting technologies, which convert mechanical vibration energy to electrical energy, have been getting attention over the last decade [1]. The mainstream of this research area has been limited in harvesting milliwattscale power from ambient structural vibration with piezoelectric devices for wireless sensing and embedded computing systems [2, 3, 4, 5]. While, recently, largescale energy harvesting from vibratory structures with electromagnetic transducers has been considered as down-to-earth renewable energy source [6]. The examples of these large-scale energy harvesting applications include automotive suspensions [7], railway systems [8], wave-excited offshore structures [9], and wind-excited high-rise buildings [10]. These references have shown that electromagnetic transducers enable watt- to kilowatt scale energy harvesting.

One of the major challenges of this technology concerns the efficient extraction and transmission of power from a transducer to a storage device such as supercapacitor or rechargeable battery. It was shown that theoretically, the rate of power conversion can be greatly improved through the use of power-electronic switching converters, which can impose static or dynamic relationships between the voltage and currents of the transducers. The currents in these converters can be controlled by operating the converter's transistors such as switches, which open and close circuit paths. Efficient conversion circuit and control systems for energy harvesting have been sought and investigated by a number of researchers $[11,12,13]$.

Meanwhile, in civil engineering, to increase energy absorption performance in structures subject to earthquake loadings, tuned viscous inertial mass dampers have been proposed $[14,15]$. The tuned viscous inertial mass damper is composed of a rotational mass damper and a supporting spring parts. The rotational mass damper part consists of a ball screw mechanism, a rotating mass, and a viscous material. The ball screw mechanism is employed to convert linear motion to rotational behavior. Then a rotary inertial mass effect is produced by rotating 
(a)

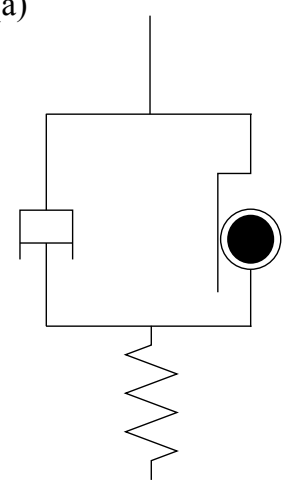

(b)

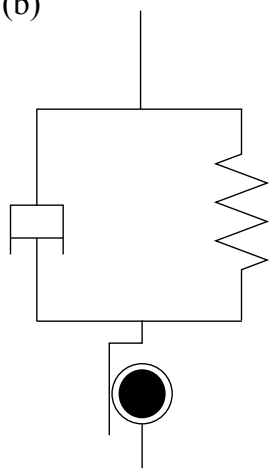

Figure 1: Damper models: (a) Tuned viscous inertial mass damper (b) Tuned inerter damper

the mass and an amplified equivalent mass effect is obtained. The force produced by the inertial mass is proportional to the relative acceleration between both ends, which is defined as inerter in [16]. This rotational mass damper is connected to a structure through the supporting spring, whose stiffness is tuned so that the rotational viscous mass damper absorbs input energy to the structure effectively. In this system, the amplified equivalent mass and the damping part are connected in parallel and the spring is arranged in series with them as depicted in Fig. 1 (a). For vibration suppression in civil structures, [17] proposes tuned inerter dampers (TID), in which the control force is generated by combining a flywheel and a gear. The mechanical model of the TID is illustrated in Fig. 1(b), schematically. The difference from the model of the tuned viscous inertial mass damper is that the spring and damping elements are arranged in parallel and the inerter part is connected in series with the spring and damping part. The equivalent mechanism called electromagnetic tuned inerter damper (E-TID) is realized in [18] with an electromagnetic device and it is shown that the E-TID is able to be operated on the self-powered energy.

This paper employs the tuned inertial mass effect and investigates the potential of a energy harvester which consists of a tuned inertial mass electromagnetic transducer (TIMET) and a single-degree-of-freedom (SDOF) oscillator. The TIMET is a device combining the electromagnetic transducer [19] and the tuned viscous inertial mass damper [14]. Both the systems can be realized through one ball screw mechanism at the same time, which makes the proposed device simple and compact. Thus the TIMET can be considered as a promising device from the view point of energy harvesting. To verify the effectiveness of the proposed device, three algorithms proposed in the literature for harvesting energy are ap- 
(a)

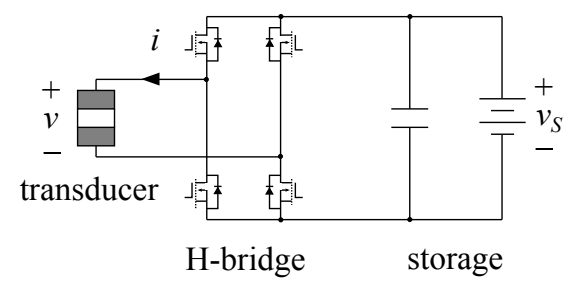

(b)

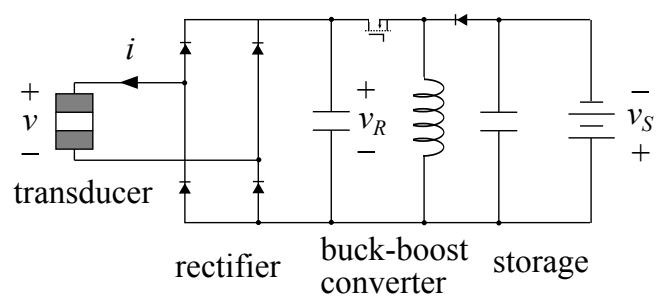

Figure 2: (a) Bi-directional converter (b) Single-directional converter

plied, i.e., static admittance (SA) control, performance-guaranteed (PG) control [20], and LQG optimal control [21].

To implement these controllers, appropriate converters need to be connected to the transducer such as a single-directional converter for the SA and the PG controllers and a bi-directional converter for the LQG controller. These converters are used to control the input current to the transducer. The bi-directional converter can be realized by using an H-bridge with four MOSFETs as illustrated in Fig. 2(a) and it enables two-way power flow. However, when the switching of a MOSFET is controlled via high-frequency pulse-width modulation (PWM), an unavoidable parasitic power loss happens due to the switching energy [22]. While, although the single-directional converter restricts the power flow, PWM operates on only a single MOSFET, so generally, the total amount of parasitic loss in single-directional converters become less than bi-directional cases.

In this paper, first, the mechanism of the proposed device is described and the mathematical models of the energy harvester with the TIMET and disturbance are developed. Then an optimization technique for the static admittance and the stiffness of the supporting spring to maximize the generated power on the proposed device subject to stochastic disturbances is introduced. And the algorithms of the PG control and the LQG optimum control are reviewed briefly. Subsequently, results calculated from numerical simulations are presented. Conclusions obtained from this study then follow.

Note that in this paper for any matrix $\mathbf{Z} \in \mathbb{R}^{n \times n}$ and $n \in \mathbb{N}, \mathbf{Z}>0$ and $\mathbf{Z} \geq 0$ represent positive definite and positive semidefinite, respectively.

\section{Tuned Inertial Mass Electromagnetic Transducer}

We begin by reviewing the electromagnetic transducer introduced in [19], which is designed to convert mechanical energy to electrical energy and vice 
(a)

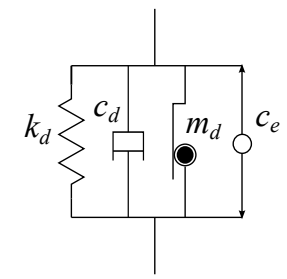

(c)

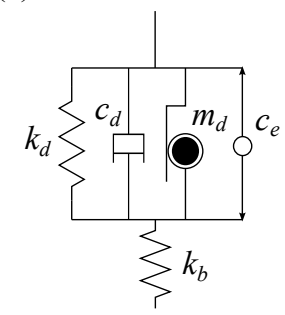

(b)

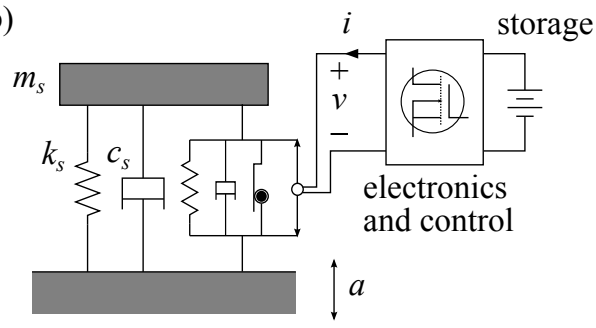

(d)

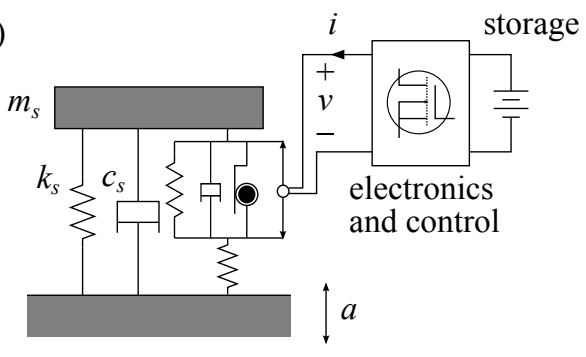

Figure 3: Models: (a) Electromagnetic transducer (b) SDOF energy harvester with an electromagnetic transducer (c) Tuned inertial mass electromagnetic transducer (TIMET) (D) SDOF energy harvester with a TIMET.

versa. The mechanical model used in [20] is illustrated in Fig. 3 (a). The electromagnetic transducer consists of a ball screw mechanism and a permanent magnet synchronous machine and has unavoidable small equivalent mass $m_{d}$, damping $c_{d}$, stiffness $k_{d}$, and electromechanical coupling $c_{e}$. The energy harvesting system consisting of the transducer embedded within a SDOF oscillator is shown in Fig. 3 (b). The SDOF oscillator has mass $m_{s}$, damping $c_{s}$, and stiffness $k_{s}$.

To increase energy absorbing capability, the energy harvester employing a TIMET is proposed in this section. In a similar way to the tuned viscous inertial mass damper introduced in the introduction, a linear spring whose stiffness is $k_{b}$ is installed in series with the electromagnetic transducer part and a larger equivalent mass is used in the proposed TIMET as shown in Fig. 3 (c). The amplified equivalent mass effect can be realized through a ball screw mechanism easily with a relatively small physical mass. The proposed energy harvester is designed by combining the TIMET with a SDOF oscillator system as illustrated in Fig. 3 (d).

The equation of motion of the proposed energy harvester is derived as follows. If $x$ is the displacement relative to the ground of the SDOF oscillator and $x_{d}$ is the displacement of the equivalent mass. Then the equation of equilibrium of the oscillator would be

$$
m_{s} \ddot{x}+c_{s} \dot{x}+k_{s} x=-m_{s} a-f_{b}
$$


where $a$ is the base acceleration and $f_{b}$ is the force from the supporting stiffness given by

$$
f_{b}=k_{b}\left(x-x_{d}\right)
$$

and the one of the transducer part becomes

$$
m_{d} \ddot{x}_{d}+c_{d} \dot{x}_{d}+k_{d} x_{d}=c_{e} i+f_{b}
$$

where $i$ is the current into the transducer. Note that the stiffness of the supporting spring must be positive, thus $k_{b}$ is constrained by

$$
k_{b} \in(0, \infty)
$$

If we define $\mathbf{x}_{E}=\left[\begin{array}{ll}x & x_{d}\end{array}\right]^{T}$, these two equations of motion for the energy harvester with the TIMET are expressed, in matrix form, as

$$
\mathbf{M}_{E} \ddot{\mathbf{x}}_{E}+\mathbf{C}_{E} \dot{\mathbf{x}}_{E}+\mathbf{K}_{E} \mathbf{x}_{E}=\mathbf{E}_{E} i+\mathbf{F}_{E} f_{b}+\mathbf{G}_{E} a
$$

where

$$
\begin{array}{rlrl}
\mathbf{M}_{E} & =\left[\begin{array}{cc}
m_{s} & 0 \\
0 & m_{d}
\end{array}\right], & \mathbf{C}_{E}=\left[\begin{array}{cc}
c_{s} & 0 \\
0 & c_{d}
\end{array}\right], & \mathbf{K}_{E}=\left[\begin{array}{cc}
k_{s} & 0 \\
0 & k_{d}
\end{array}\right], \\
\mathbf{E}_{E}=\left[\begin{array}{c}
0 \\
c_{e}
\end{array}\right], & \mathbf{F}_{E}=\left[\begin{array}{c}
-1 \\
1
\end{array}\right], & \mathbf{G}_{E}=\left[\begin{array}{c}
-m_{s} \\
0
\end{array}\right]
\end{array}
$$

\section{Energy Harvesting Problem for the TIMET}

To investigate the potential power generation on the energy harvester with the TIMET controlled by the SA, PG, and LQG optimal laws, the state-space forms of the equation of motion of the device and the input disturbance are developed. And the objective function for the energy harvesting performance is defined. Then three algorithms applied in this paper are introduced briefly.

\subsection{Harvester and disturbance modeling}

The equation of motion of the proposed vibration energy harvester derived in Section 2 can be rewritten in state-space form, by defining $\mathbf{x}_{h}=\left[\begin{array}{ll}\mathbf{x}_{E}^{T} & \dot{\mathbf{x}}_{E}^{T}\end{array}\right]^{T}$, as

$$
\dot{\mathbf{x}}_{h}(t)=\mathbf{A}_{h} \mathbf{x}_{h}(t)+\mathbf{B}_{h} i(t)+\mathbf{F}_{h} f_{b}+\mathbf{G}_{h} a(t)
$$


where

$$
\begin{aligned}
\mathbf{A}_{h} & =\left[\begin{array}{cc}
\mathbf{0} & \mathbf{I} \\
-\mathbf{M}_{E}^{-1} \mathbf{K}_{E} & -\mathbf{M}_{E}^{-1} \mathbf{C}_{E}
\end{array}\right], & \mathbf{B}_{h} & =\left[\begin{array}{c}
\mathbf{0} \\
\mathbf{M}_{E}^{-1} \mathbf{E}_{E}
\end{array}\right], \\
\mathbf{F}_{h} & =\left[\begin{array}{c}
\mathbf{0} \\
\mathbf{M}_{E}^{-1} \mathbf{F}_{E}
\end{array}\right], & & \mathbf{G}_{h}=\left[\begin{array}{c}
\mathbf{0} \\
\mathbf{M}_{E}^{-1} \mathbf{G}_{E}
\end{array}\right]
\end{aligned}
$$

and the voltage of the transducer is assumed to be the output of the system as

$$
v=\mathbf{C}_{h} \mathbf{x}_{h}(t)
$$

where

$$
\mathbf{C}_{h}=\left[\begin{array}{llll}
0 & 0 & 0 & c_{e}
\end{array}\right]
$$

We assume that the disturbance acceleration $a(t)$ is filtered white noise and its power spectral density equals to

$$
S_{a}(\omega)=\left|\frac{j q \omega}{-\omega^{2}+2 j \zeta_{a} \omega_{a} \omega+\omega_{a}^{2}}\right|^{2}
$$

where $j$ is the imaginary unit (i.e., $j^{2}=-1$ ), $\omega_{a}$ is the center of the passband of $a(t)$ and $\zeta_{a}$ determines the spread of its frequency content. For such a process, it is straightforward to represent the disturbance dynamics by a two-dimensional state-space of the form

$$
\begin{aligned}
\dot{\mathbf{x}}_{a}(t) & =\mathbf{A}_{a} \mathbf{x}_{a}(t)+\mathbf{B}_{a} w(t) \\
a(t) & =\mathbf{C}_{a} \mathbf{x}_{a}(t)
\end{aligned}
$$

where

$$
\mathbf{A}_{a}=\left[\begin{array}{cc}
0 & 1 \\
-\omega_{a}^{2} & -2 \zeta_{a} \omega_{a}
\end{array}\right], \quad \mathbf{B}_{a}=\left[\begin{array}{c}
0 \\
2 \sigma_{a} \sqrt{\zeta_{a} \omega_{a}}
\end{array}\right], \quad \mathbf{C}_{a}=\left[\begin{array}{ll}
0 & 1
\end{array}\right]
$$

and $w(t)$ is a white noise with spectral intensity equal to 1 . The parameter $q$ in Eq. (13) is adjusted such that $a(t)$ has a consistent standard deviation of $\sigma_{a}$, i.e.,

$$
\sigma_{a}=\sqrt{\frac{1}{2 \pi} \int_{-\infty}^{\infty} S_{a}(\omega) d \omega}
$$

This allows us to compare energy harvesting performances from disturbances of varying spectral content but equal intensity. Note that when $\zeta_{a}$ is close to 0 , the 
disturbance becomes narrowband random vibration. Especially, as $\zeta_{a} \rightarrow 0$, the disturbance approaches to a sine wave. While, when $\zeta_{a}$ is a large value, the disturbance contains broadband frequency contents.

Combining the harvester and disturbance dynamics yields an augmented system given by

$$
\begin{aligned}
\dot{\mathbf{x}}(t) & =\mathbf{A x}(t)+\mathbf{B} i(t)+\mathbf{F} f_{b}+\mathbf{G} w(t) \\
v & =\mathbf{C x}(t)
\end{aligned}
$$

where the augmented matrices $\mathbf{A}, \mathbf{B}, \mathbf{F}, \mathbf{G}$, and $\mathbf{C}$ are

$$
\begin{array}{rlrl}
\mathbf{A} & =\left[\begin{array}{cc}
\mathbf{A}_{h} & \mathbf{G}_{h} \mathbf{C}_{a} \\
\mathbf{0} & \mathbf{A}_{a}
\end{array}\right], & \mathbf{B}=\left[\begin{array}{c}
\mathbf{B}_{h} \\
\mathbf{0}
\end{array}\right], & \mathbf{F}=\left[\begin{array}{c}
\mathbf{F}_{h} \\
\mathbf{0}
\end{array}\right], \\
\mathbf{G}=\left[\begin{array}{c}
\mathbf{0} \\
\mathbf{B}_{a}
\end{array}\right], & \mathbf{C}=\left[\begin{array}{ll}
\mathbf{C}_{h} & \mathbf{0}
\end{array}\right]
\end{array}
$$

and the resultant augmented state vector is $\mathbf{x}=\left[\begin{array}{ll}\mathbf{x}_{h}^{T}(t) & \mathbf{x}_{a}^{T}(t)\end{array}\right]^{T}$.

\subsection{Energy harvesting objective}

To assess the energy harvesting capability of the proposed device, the energy harvesting objective for the system in Eqs. (18) and (19) is needed. In this paper, the power delivered to storage is defined as the power extracted by the harvester minus the transmission losses in the transducer and power electronics circuitry, i.e.,

$$
P_{S}(t)=-i(t) v(t)-P_{d}(t)
$$

where $P_{d}(t)$ is the transmission dissipation. Generally, the dissipation $P_{d}(t)$ is quite complicated, and it depends not only on the electronic hardware used to realize the controller but also on the manner in which this hardware is operated such as its switching frequency, bus voltage, and MOSFET gating voltage. However, for simplicity, the assumption that the power dissipated in the electronics is resistive, i.e.,

$$
P_{d}(t)=R i^{2}(t)
$$

is used in this paper as in [20]. Provided these assumptions, the energy harvesting objective can be defined as the expectation of Eq. (22), i.e.,

$$
\bar{P}_{g}=-\mathscr{E}\left\{\left[\begin{array}{c}
\mathbf{x} \\
i
\end{array}\right]^{T}\left[\begin{array}{cc}
\mathbf{0} & \frac{1}{2} \mathbf{C}^{T} \\
\frac{1}{2} \mathbf{C} & R
\end{array}\right]\left[\begin{array}{c}
\mathbf{x} \\
i
\end{array}\right]\right\}
$$

The objective of this section is to increase energy harvesting performance expressed by Eq. (24) through various algorithms and converter systems. 


\subsection{Admittance constraint for the single-directional converter}

For the SA and PG controllers, we refer to the single-directional converter used in [20]. In this case, the input current to the transducer $i(t)$ is expressed by

$$
i(t)=-Y(t) v(t)
$$

where $i(t)$ is the current into the transducer, $v(t)$ is the transducer voltage, $Y(t)$ has units of admittance, and in addition, the constraint

$$
Y(t) \geq 0
$$

is required for the one-way power directionality. We assume further that a buckboost converter controlled via high-frequency PWM switching of a single MOSFET as shown in Fig. 2 (b) is employed and that the converter is operated in discontinuous conduction mode (DCM). This is because these assumptions have two advantages. First, in terms of the rectifier of voltage $V_{R}$, the input impedance of the converter can be approximated by resistance at frequencies well below the switching frequency of the converter [22]. Second, the input impedance is barely affected by the behavior of storage voltage $V_{S}$.

The desired admittance values to control the input current to the transducer is realized by changing the duty cycle of the MOSFET. However, the admittance value is bounded from above by $Y^{\max }$, which is determined by the limit value of the duty cycle for the DCM. The value of $Y^{\max }$ can be calculated using the known parameters of the power electronic converter such as inductance, switching frequency, etc, which is beyond the scope of this paper. Also, as mentioned, the admittance is restricted by Eq. (26). Therefor these two restrictions result in the following constraint

$$
Y \in\left[0, Y^{\max }\right]
$$

Through the duty cycle of the MOSFET connecting with a comparator, the admittance value can be controlled. For example, the duty cycle for the static admittance can be obtained by connecting a passive circuit containing two parallel resistors to the non-inverting input of the comparator. The details of the method to realize the constant admittance can be found in [23, 24]. While, for time-varying admittance as used in the PG algorithm, the duty cycle is controlled on a real-time basis by employing a sawtooth function with a specified frequency [20].

\subsection{Optimal static admittance and spring stiffness}

To apply the SA control to the TIMET, unlike the traditional electromagnetic transducer case as shown in Fig. 3 (a), the stiffness of the supporting spring $k_{b}$ as 
well as the constant admittance $Y_{c}$ need to be determined. The method to explore the optimal values for these parameters to maximize the generated power given by Eq. (24) is introduced here.

The input current to the transducer and the force from the supporting spring are expressed as functions of the state variable $\mathbf{x}$, i.e.,

$$
i(t)=-Y_{c} \mathbf{C x}(t)
$$

and

$$
f_{b}(t)=k_{b} \mathbf{T} \mathbf{x}(t)
$$

where $\mathbf{T}=\left[\begin{array}{llllll}1 & -1 & 0 & 0 & 0 & 0\end{array}\right]$. Substituting Eqs. (28) and (29) into Eq. (18) yields the closed-loop dynamics having the form

$$
\dot{\mathbf{x}}(t)=\left(\mathbf{A}-Y_{c} \mathbf{B C}+k_{b} \mathbf{F T}\right) \mathbf{x}(t)+\mathbf{G} w(t)
$$

For this linear time-invariant system, the optimal values for $Y_{c}$ and $k_{b}$ are obtained as follows.

For any time-invariant $Y_{c}$ and $k_{b}$ satisfying Eqs. (27) and (4), it is a standard result (see [25], for example) that the power generation objective can be written as

$$
\bar{P}_{g}^{S A}=-\operatorname{tr}\left[\mathbf{G}^{T} \mathbf{S}_{\mathrm{SA}} \mathbf{G}\right]
$$

where $\mathbf{S}_{\mathrm{SA}}=\mathbf{S}_{\mathrm{SA}}^{T}<0$ is the solution to the Lyapunov equation

$$
\left(\mathbf{A}-Y_{c} \mathbf{B C}+k_{b} \mathbf{F T}\right)^{T} \mathbf{S}_{\mathrm{SA}}+\mathbf{S}_{\mathrm{SA}}\left(\mathbf{A}-Y_{c} \mathbf{B C}+k_{b} \mathbf{F T}\right)+\mathbf{C}^{T}\left(-Y_{c}+Y_{c}^{2} R\right) \mathbf{C}=\mathbf{0}
$$

As mentioned in Section 3.3, the value of $Y^{\max }$ is affected by the MOSFET. Thus, for an ideal system, $Y^{\max }$ must be less than or equal to $1 / R$, so the last term on the left-hand side of Eq. (32) is negative-semidefinite for all $Y_{c}$. Thus, since $\mathbf{A}-Y_{c} \mathbf{B C}+k_{b} \mathbf{F T}$ is asymptotically stable, the definiteness of $\mathbf{S}_{\mathrm{SA}}$ is assured by Lyapunov's second theorem [26].

The above conditions can be rewritten in the form of a bilinear matrix inequality (BMI) problem with the products $\left(\mathbf{A}-Y_{c} \mathbf{B C}+k_{b} \mathbf{F T}\right)^{T} \mathbf{S}_{\mathrm{SA}}$ comprising the bilinear terms in the expressions. This leads to the following theorem. In the interest of brevity, the theorem is not proved here, however, this is analogous to other standard techniques for convex analysis of Lyapunov equations [27].

THEOREM 1. Let $\bar{P}_{g m}$ be an expected power generation of a specific value. Then for any $Y_{c} \in\left[0, Y^{\mathrm{max}}\right]$ and $k_{b} \in(0, \infty), \bar{P}_{g}>\bar{P}_{g m}$ if and only if $\exists \Sigma_{S A}=\Sigma_{S A}^{T}<0$, such that

$$
-\operatorname{tr}\left[\mathbf{G}^{T} \Sigma_{S A} \mathbf{G}\right]>\bar{P}_{g m}
$$




$$
\left[\begin{array}{cc}
\mathbf{A}_{c l}^{T} \Sigma_{S A}+\Sigma_{S A} \mathbf{A}_{c l}-\mathbf{C}^{T} Y_{c} \mathbf{C} & Y_{c} \mathbf{C}^{T} \\
Y_{c} \mathbf{C} & R^{-1}
\end{array}\right]>0
$$

where $\mathbf{A}_{c l}=\mathbf{A}-Y_{c} \mathbf{B C}+k_{b} \mathbf{F T}$

The objective is to maximize $\bar{P}_{g m}$, subject to constraints Eqs. (27), (4), (33), (34), and $\Sigma_{\mathrm{SA}}<0$. We note that the fact that $\mathbf{A}-Y_{c} \mathbf{B C}+k_{b} \mathbf{F T}$ is known to be asymptotically stable for all $Y_{c}$ and $k_{b}$ satisfying Eqs. (27) and (4) is exploited; if this were not the case, then it could not be assumed that Eq. (34) ensures $\Sigma_{S A}>\mathbf{S}_{S A}$, which is necessary for Eq. (33) to conservatively enforce $\bar{P}_{g}^{S A} \geq \bar{P}_{g m}$.

BMI-constrained optimizations are nonconvex, which can be converted to convex optimizations (through a change of coordinates) only in very special cases. Unfortunately, in this particular problem, a technique to do this is not known. Thus nonconvex optimization techniques must be used. Results in this paper were obtained using convex over-bounding techniques, as originally proposed by [28] and [29]. In this technique, first, the bilinear terms such as $Y_{c} \Sigma_{\mathrm{SA}}$ and $k_{b} \Sigma_{\mathrm{SA}}$ in Eq. (34) are linearized. Then, to maximize $\bar{P}_{g m}$, the values of $\Sigma_{\mathrm{SA}}, Y_{c}$, and $k_{b}$ are found in the constrained feasible regions iteratively. The details of these techniques are not described in this paper. However, the outline of the method can be found in [30].

\subsection{Performance-guaranteed control with a single-directional converter}

For comparison, the efficacy of the TIMET controlled by the PG algorithm proposed in [20] is examined. This algorithm is operated with a single-directional converter as shown in Fig. 2 (b). Then the admittance $Y$ becomes a function of time varying within the range of Eq. (27) so that the generated power must be larger than the SA case, i.e.,

$$
\bar{P}_{g}^{\mathrm{PG}} \geq \bar{P}_{g}^{\mathrm{SA}}
$$

where $\bar{P}_{g}^{\mathrm{PG}}$ represents the generated power by the PG control.

To implement this algorithm, $k_{b}^{*}$ obtained for the SA control is used for the stiffness for the supporting spring. Thus, the augmented system Eq. (18) for the TIMET is modified as

$$
\begin{aligned}
\dot{\mathbf{x}}(t) & =\overline{\mathbf{A}} \mathbf{x}(t)+\mathbf{B} i(t)+\mathbf{G} w(t) \\
& =(\overline{\mathbf{A}}-Y(t) \mathbf{B C}) \mathbf{x}+\mathbf{G} w(t)
\end{aligned}
$$

where

$$
\overline{\mathbf{A}}=\left[\begin{array}{cc}
\overline{\mathbf{A}}_{h} & \mathbf{G}_{h} \mathbf{C}_{a} \\
\mathbf{0} & \mathbf{A}_{a}
\end{array}\right], \quad \overline{\mathbf{A}}_{h}=\left[\begin{array}{cc}
\mathbf{0} & \mathbf{I} \\
-\mathbf{M}_{E}^{-1} \overline{\mathbf{K}}_{E} & -\mathbf{M}_{E}^{-1} \mathbf{C}_{E}
\end{array}\right]
$$


and

$$
\overline{\mathbf{K}}_{E}=\left[\begin{array}{cc}
k_{s}+k_{b}^{*} & -k_{b}^{*} \\
-k_{b}^{*} & k_{d}+k_{b}^{*}
\end{array}\right]
$$

The PG control is described by the next theorem.

THEOREM 2. For the system in Eq. (36), let $Y_{c}^{*}$ be the optimal static admittance. Then the input current for the PG control is given by

$$
i(t)= \begin{cases}i_{u}(t) & : i_{u} v+i_{u}^{2} / Y^{\max } \leq 0 \\ 0 & : i_{u} v+i_{u}^{2} / Y^{\max }>0 \text { and } i_{u} v>0 \\ -Y^{\max } v(t) & : \text { otherwise }\end{cases}
$$

where

$$
i_{u}=-\frac{1}{R}\left(\mathbf{B}^{T} \mathbf{S}_{P G}+\frac{1}{2} \mathbf{C}\right) \mathbf{x}
$$

and $\mathbf{S}_{P G}=\mathbf{S}_{P G}^{T}<0$ is the solution to the Lyapunov equation

$$
\left(\overline{\mathbf{A}}-Y_{c}^{*} \mathbf{B C}\right)^{T} \mathbf{S}_{P G}+\mathbf{S}_{P G}\left(\overline{\mathbf{A}}-Y_{c}^{*} \mathbf{B C}\right)+\mathbf{C}^{T}\left(-Y_{c}^{*}+Y_{c}^{* 2} R\right) \mathbf{C}=\mathbf{0}
$$

And the energy harvesting performance obtained by this input current becomes

$$
\bar{P}_{g}^{P G}=\bar{P}_{g}^{S A}+R \mathscr{E}\left\{\left(i_{u}+Y_{c}^{*} v\right)^{2}-\left(i_{u}-i\right)^{2}\right\}
$$

which satisfies Eq. (35).

Note that $\mathbf{S}_{\mathrm{PG}}=\mathbf{S}_{\mathrm{SA}}$ because Eq. (41) is nothing but Eq. (32). The details and proof of the PG control is provided in [20]. This controller can be realized with a single-directional converter and the energy harvesting performance of the SA controller is guaranteed. However, to implement this controller, in general, the full-state measurements is required.

\subsection{LQG optimal control with a bi-directional converter}

Finally, the energy harvesting performance of the TIMET by the LQG optimal control is investigated. As in the case of the PG control, $k_{b}^{*}$ obtained for the SA control is used for the stiffness of the supporting spring $k_{b}$. We assume here that an unconstrained bi-directional converter is employed such that the $\mathrm{H}$-bridge can control the current $i(t)$ with high enough bandwidth. In this case, it is shown that the LQG optimal control provides the causal limit on power generation in [21]. The controller and the energy harvesting performance are summarized in the next theorem. 
THEOREM 3. The optimal energy harvesting current is given by the linear state feedback relationship

$$
i=-\frac{1}{R}\left\{\mathbf{B}^{T} \mathbf{S}_{L Q G}+\frac{1}{2} \mathbf{C}\right\} \mathbf{x}
$$

where $\mathbf{S}_{L Q G}=\mathbf{S}_{L Q G}^{T}<0$ is the solution to the nonstandard Riccati equation

$$
\begin{aligned}
\left(\overline{\mathbf{A}}-\frac{1}{2 R} \mathbf{B C}\right)^{T} \mathbf{S}_{L Q G}+\mathbf{S}_{L Q G}\left(\overline{\mathbf{A}}-\frac{1}{2 R} \mathbf{B C}\right) & \\
& -\frac{1}{R}\left(\mathbf{S}_{L Q G} \mathbf{B B}^{T} \mathbf{S}_{L Q G}+\frac{1}{4} \mathbf{C}^{T} \mathbf{C}\right)=\mathbf{0}
\end{aligned}
$$

By this controller, the optimal average power generation is

$$
\bar{P}_{g}^{L Q G}=-\mathbf{G}^{T} \mathbf{S}_{L Q G} \mathbf{G}
$$

The poof of this theorem is not provided here, however it can be found in [21]. Generally, for this controller, all the states need to be measured as well.

\section{Numerical Simulations}

To validate the efficacy of the proposed energy harvester with the TIMET, numerical simulation studies are carried out. To decide realistic parameter values for the proposed device, we referred to the values for the electromagnetic transducers which are derived experimentally and used in $[19,20]$. Then the results are compared with the electromagnetic transducer case studied in [20]. The natural frequency of the SDOF oscillator used in this study is $\sqrt{k_{s} / m_{s}}=3.16 \mathrm{rad} / \mathrm{s}$. To investigate the effect of the inertial mass of the TIMET, two different equivalent masses, i.e., (a) $20 \mathrm{~kg}$ (same as the electromagnetic transducer) and (b) $400 \mathrm{~kg}$, are employed. Table 1 summarizes the parameter values used in this study. Because

the performance of the PG controller does not have a closed-form solution, $\bar{P}_{g}^{\mathrm{PG}}$ is obtained as an approximate stationary solution through simulation for $3000 \mathrm{~s}$ at a sample of rate of $100 \mathrm{~Hz}$ as in [20]. The initial condition of Eq. (14) is set to 0. The natural period of the oscillator is about $1 \mathrm{~s}$, so $3000 \mathrm{~s}$ simulation is considered to be long enough to ignore the effect of the initial condition of the filter. The upper limit for $k_{b}$ is set to $3000 \mathrm{kN} / \mathrm{m}$ because of implementing the optimization problems for the SA controller. This value is large enough for the system to consider the supporting spring as a rigid body. These numerical simulations are implemented within MATLAB. The obtained $\bar{P}_{g}$ to various $\omega_{a}$ from 2 to $5 \mathrm{rad} / \mathrm{s}$ 
Table 1: Parameter values of the electromagnetic energy harvester.

\begin{tabular}{|c|c|c|}
\hline \multirow[b]{2}{*}{ Parameter } & \multirow{2}{*}{ ET } & TIMET \\
\hline & & (b) \\
\hline$m_{s}$ & \multicolumn{2}{|c|}{$3000 \mathrm{~kg}$} \\
\hline$c_{s}$ & \multicolumn{2}{|c|}{$395 \mathrm{~N} \mathrm{~s} / \mathrm{m}$} \\
\hline$k_{s}$ & \multicolumn{2}{|c|}{$3 \times 10^{4} \mathrm{~N} / \mathrm{s}$} \\
\hline$m_{d}$ & $20 \mathrm{~kg}$ & $400 \mathrm{~kg}$ \\
\hline$c_{d}$ & \multicolumn{2}{|c|}{$575 \mathrm{~N} \mathrm{~s} / \mathrm{m}$} \\
\hline$k_{d}$ & \multicolumn{2}{|c|}{$630 \mathrm{~N} / \mathrm{m}$} \\
\hline$c_{e}$ & \multicolumn{2}{|c|}{453 N/A } \\
\hline$Y^{\max }$ & \multicolumn{2}{|c|}{$0.05 \Omega^{-1}$} \\
\hline$\sigma_{a}$ & \multicolumn{2}{|c|}{$0.18 \mathrm{~m} / \mathrm{s}^{2}$} \\
\hline
\end{tabular}

for the cases of $\zeta_{a}=0.05, \zeta_{a}=0.5$, and $\zeta_{a}=1$ are plotted in Figs. 4 through 12 . It should be noted that small $\zeta_{a}$ represents a narrowband disturbance and large $\zeta_{a}$ produces a broadband disturbance. To examine the effect of the resistance, two values are employed, i.e., $R=5 \Omega, R=20 \Omega$ for the three controllers.

The results obtained from the SA control are shown in Figs. 4 through 6. In theses figures, the ratios of the TIMET cases to the standard electromagnetic transducer cases, and the optimized values for $Y_{c}$ and $k_{b}$, which are represented by $Y_{c}^{*}$ and $k_{b}^{*}$, respectively, are plotted as well. As can be clearly seen in Figs. 4 through 6, it can be concluded that the proposed TIMET with the SA controller performs better than the general electromagnetic transducer in terms of energy harvesting performance measure. Especially, when the input disturbance is narrowband, the maximum values obtained from the TIMET (a) and (b) show huge advantages compared to the ones of the electromagnetic transducer. This results imply that if the TIMET is tuned appropriately, we can take full advantage of the capabilities of the proposed device to narrowband input. The difference between the electromagnetic transducer and TIMET (a) cases shows that the use of the supporting spring facilitates the energy harvesting performance dramatically. While, the effect of the inertial mass of the TIMET does not lead to significant differences in the maximum power generation when narrowband input is applied and the resistance $R$ is small. However, as the disturbance includes more broadband frequencies and the resistance becomes higher, the TIMET with a larger inertial mass increases the performance as shown in Figs. 5 (b) and 6 (b). Therefore we 
(a)
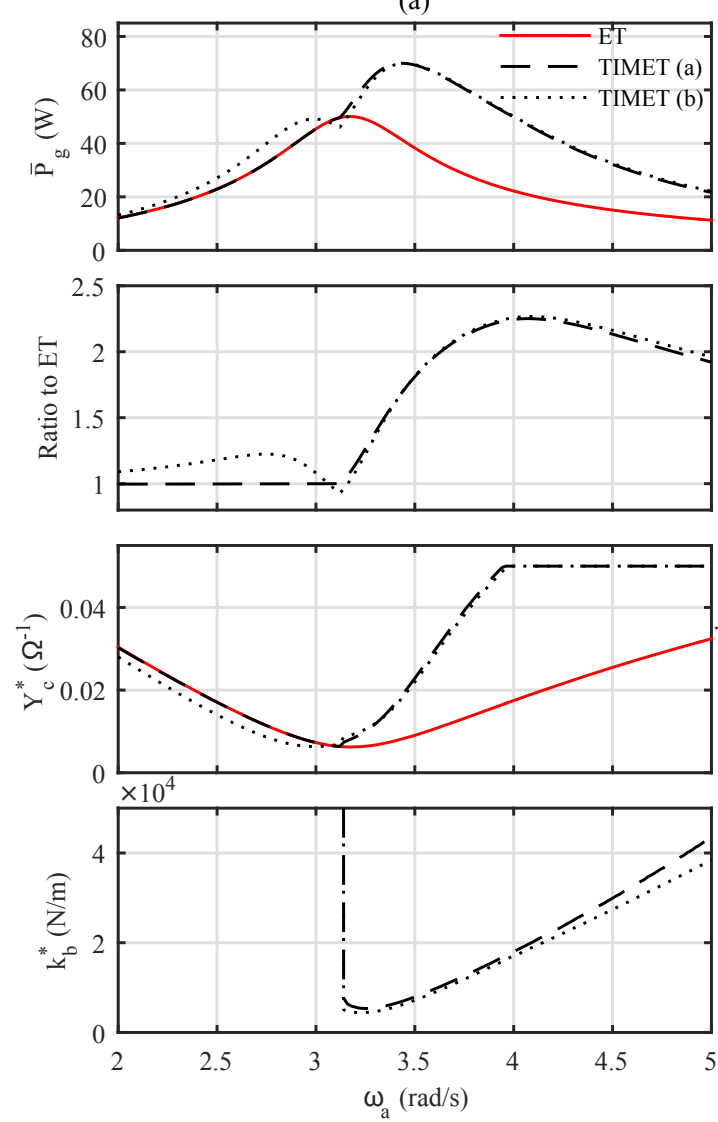

(b)
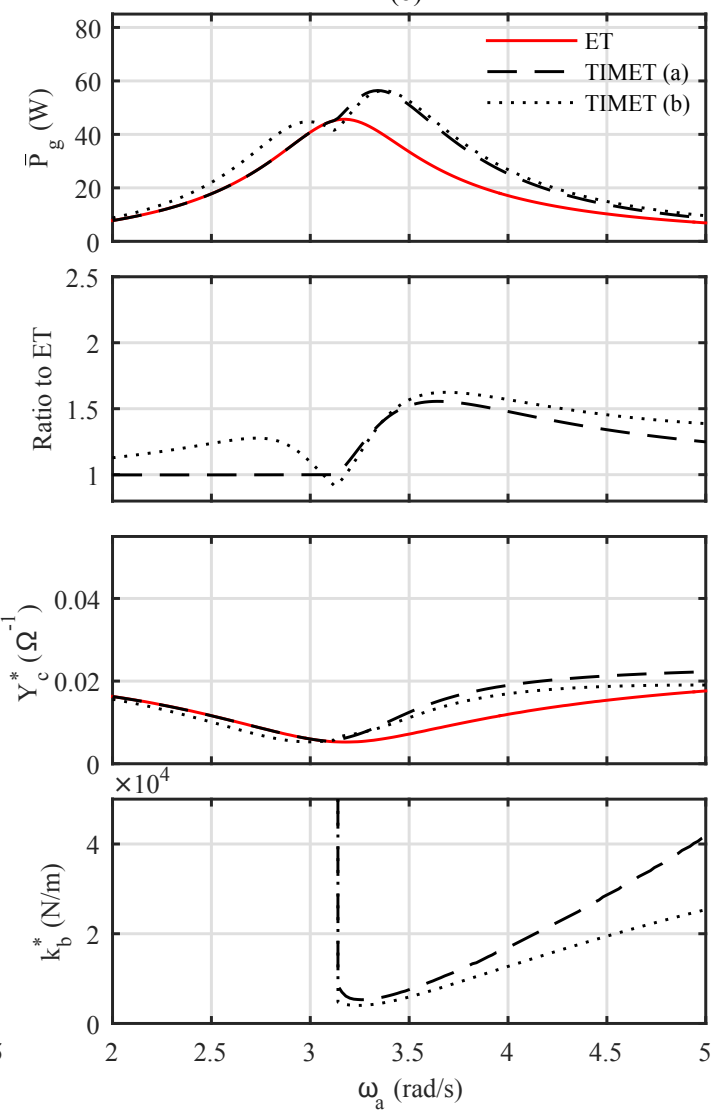

Figure 4: Comparisons of the energy harvesters obtained by the SA control for $\zeta_{a}=0.05$; (a) $R=5 \Omega$, (b) $R=20 \Omega$.

can conclude that the inertial mass provides robustness to broadband disturbances in a resistive circuit.

Figures 7 through 9 compares the results of the PG control cases. These figures provide the power generation, ratios to the electromagnetic cases, and ratios to the SA cases. For the narrowband input case as shown in Fig. 7, the advantage of the proposed device can be found in both resistance cases. However, as shown in Figs. 8 and 9, the superiority of the TIMET disappears gradually as the input frequency component spreads, especially for the low resistance case .

Similar trends to the PG cases can be observed in the LQG control cases as shown in Figs. 10 through 12, i.e., the TIMET works well to the narrowband input, while it is not advantageous to the broadband input especially when the resistance 
(a)
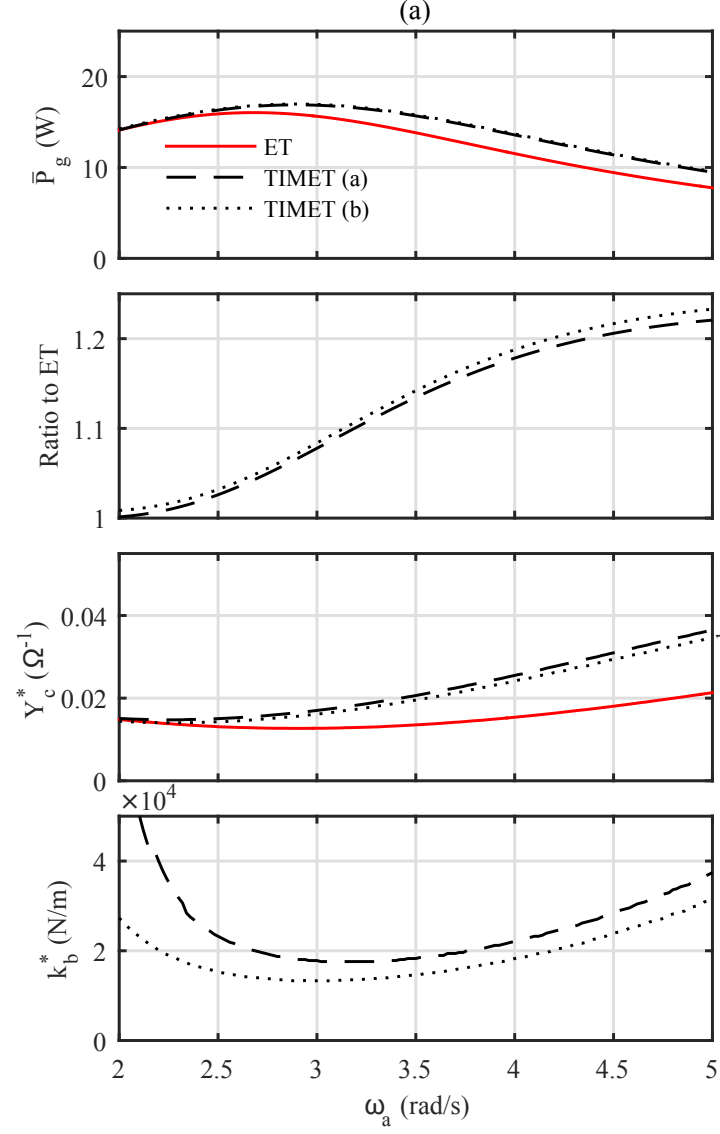

(b)
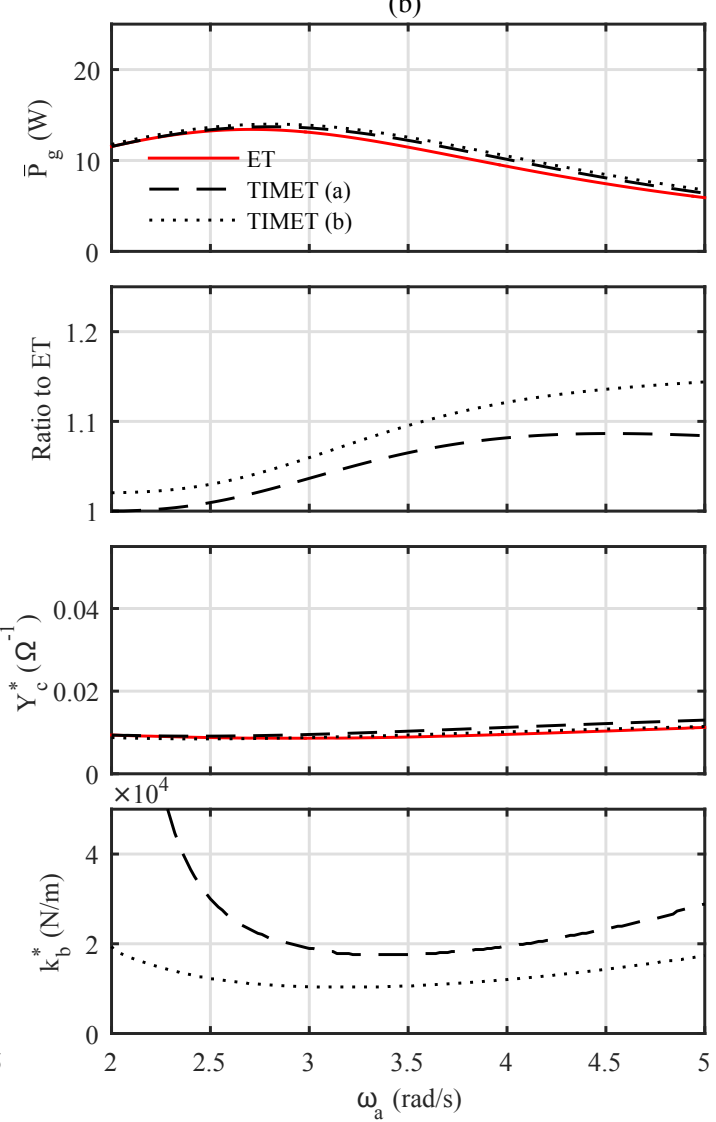

Figure 5: Comparisons of the energy harvesters obtained by the SA control for $\zeta_{a}=0.5$; (a) $R=5 \Omega$, (b) $R=20 \Omega$.

is $5 \Omega$. As can be seen in the ratios to the SA controllers, the TIMET does not exploit the features of the PG and LQG controllers as much as the electromagnetic transducer does. These results lead to the conclusion that when the PG and LQG controllers are applied, the existence of the supporting spring tuned to a certain frequency have a risk of hampering the energy harvesting performance, especially to broadband inputs.

\section{Conclusions}

The primary purposes of this paper have been to investigate the energy harvesting potential of the TIMET installed on a SDOF oscillator to stochastic disturbances. The results obtained from the numerical simulation studies showed 

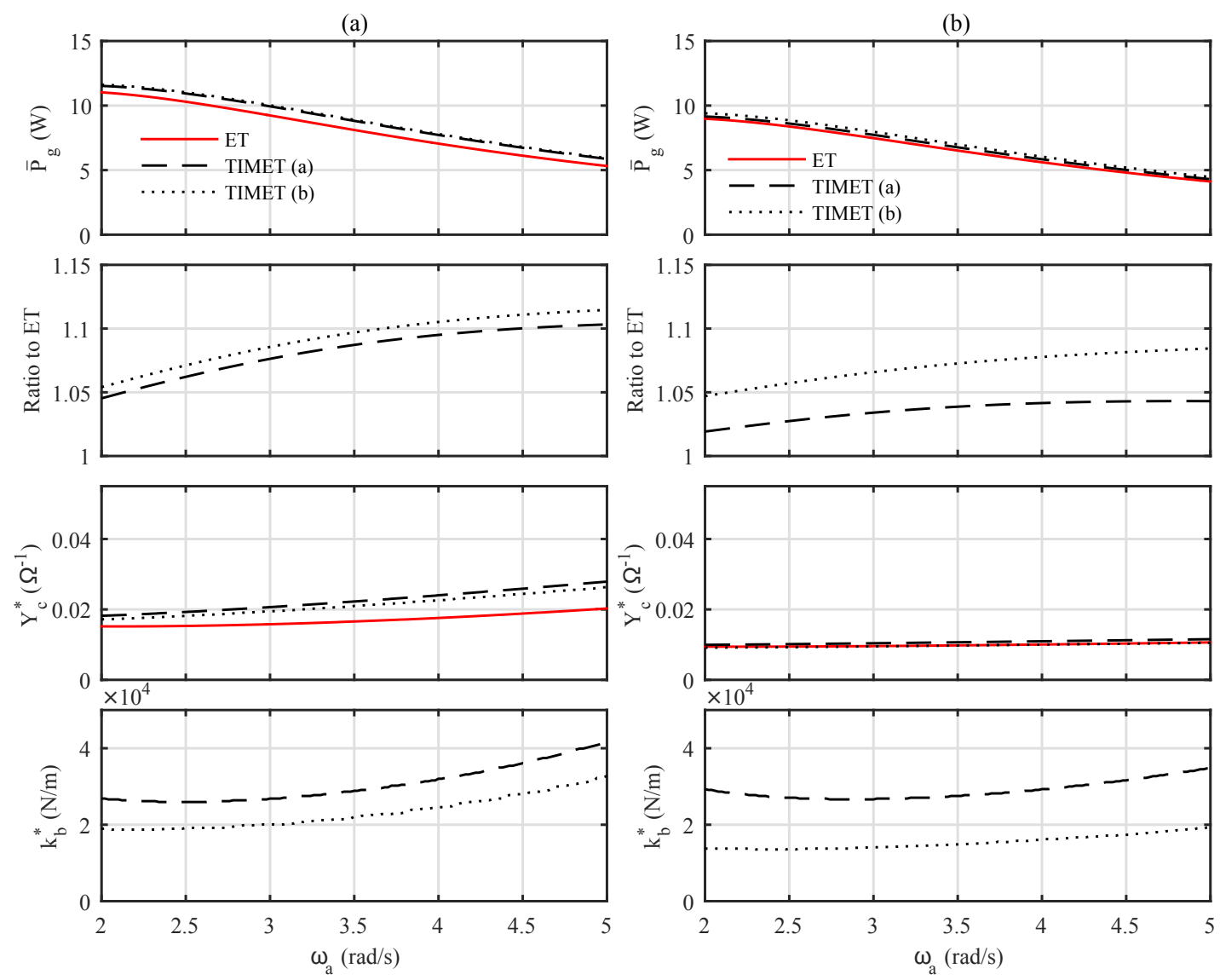

Figure 6: Comparisons of the energy harvesters obtained by the SA control for $\zeta_{a}=1$; (a) $R=5 \Omega$, (b) $R=20 \Omega$.

the effectiveness of the TIMET, especially when the SA controller which does not require full-state feedback is applied. These results lead to the conclusion that the energy harvesting performance of the electromagnetic transducer can be increased by uses of a tuned spring appropriately and a rotational mass without applying complex control algorithms which require full-state measurements such as the PG and LQG controllers. Contrary to our expectations, the maximum energy harvesting performance to narrowband inputs did not depend that much on the inertial mass effect for the three controllers.

Still a major advantage of the proposed device over the traditional electromagnetic transducer was observed when the input disturbance was narrowband. Therefore, we can expect that the TIMET works well when it is applied to civil 
(a)
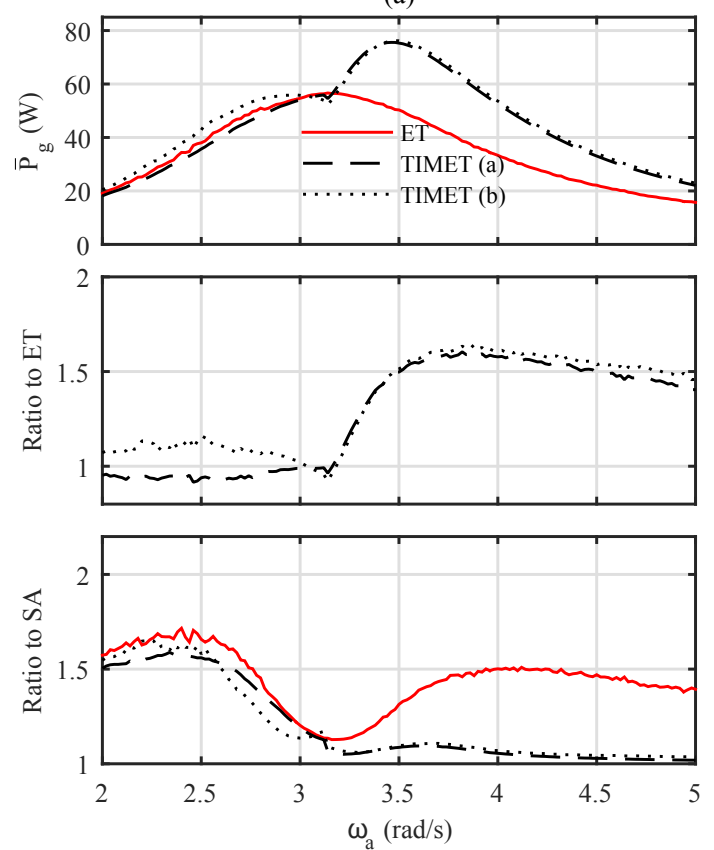

(b)
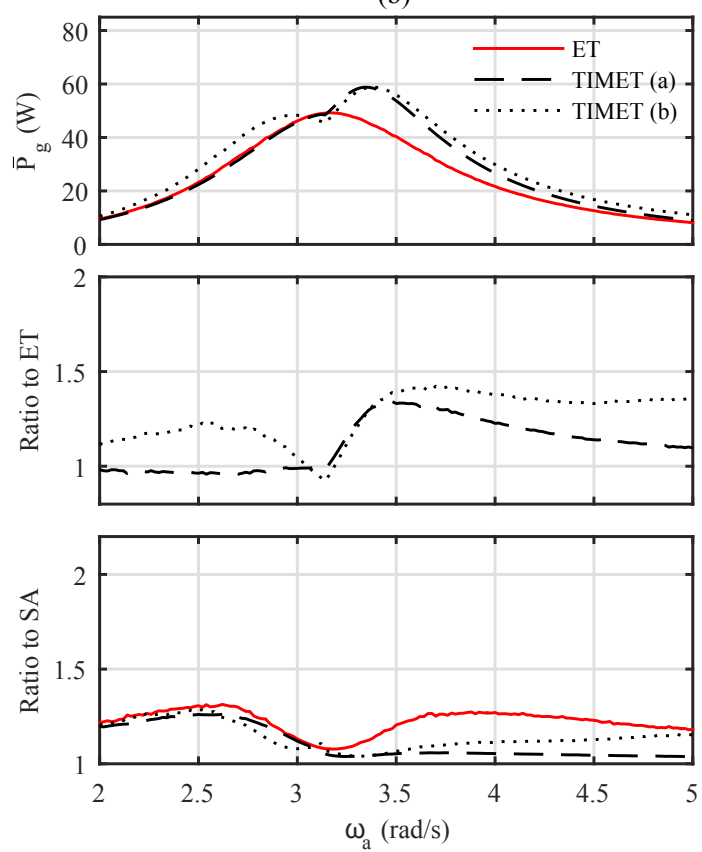

Figure 7: Comparisons of the energy harvesters obtained by the PG control for $\zeta_{a}=0.05$; (a) $R=5 \Omega$, (b) $R=20 \Omega$.

structures. This is because buildings and bridges are exposed to a variety of external disturbances such as earthquake loadings, wind forces, cars, and trains and vibrations are induced constantly. Also, in general, civil structures have a huge mass and a dominant natural frequency in a low frequency range (typically less than $10 \mathrm{~Hz}$ ). These properties are suitable for the proposed device. Thus by tuning the TIMET to the dominant frequency, vibration of civil structures can be a new renewable energy source. Hence examining the possibilities of these applications is an important subject of the future work. As another future work, experimental verification of the proposed energy harvester is required and the potential abilities of the TIMET as a self-powered control system should be investigated as well.

\section{Acknowledgements}

This research was supported by JSPS KAKENHI Grant Number 26630256. Also, the first author gratefully acknowledges support through the Grant-in-Aid for JSPS Fellows. 

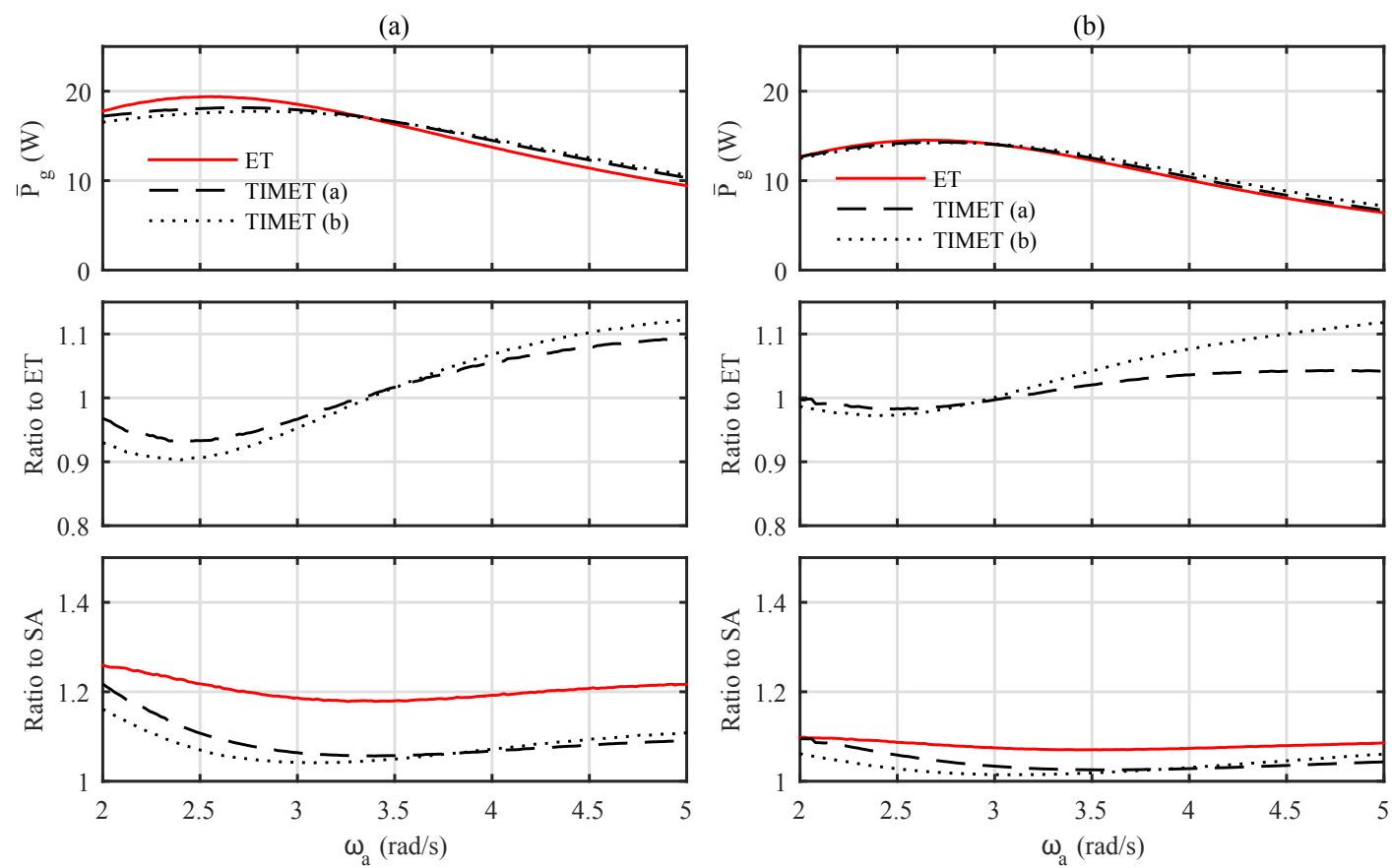

Figure 8: Comparisons of the energy harvesters obtained by the PG control for $\zeta_{a}=0.5$; (a) $R=5 \Omega$, (b) $R=20 \Omega$.

[1] S. Priya, D. Inman, Energy Harvesting Technologies, Springer US, 2008.

[2] S. Roundy, P. K. Wright, J. Rabaey, A study of low level vibrations as a power source for wireless sensor nodes, Computer Communications 26 (11) (2003) 1131 - 1144. doi:http://dx.doi.org/10.1016/S0140-3664(02)00248-7.

[3] S. P. Beeby, M. J. Tudor, N. M. White, Energy harvesting vibration sources for microsystems applications, Measurement Science and Technology 17 (12) (2006) R175.

[4] S. R. Anton, H. A. Sodano, A review of power harvesting using piezoelectric materials (2003-2006), Smart Materials and Structures 16 (3) (2007) R1.

[5] C. Knight, J. Davidson, S. Behrens, Energy options for wireless sensor nodes, Sensors 8 (12) (2008) 8037. doi:10.3390/s8128037.

[6] L. Zuo, X. Tang, Large-scale vibration energy harvesting, Journal of Intelligent Material Systems and Structures 24 (11) (2013) 1405- 
(a)
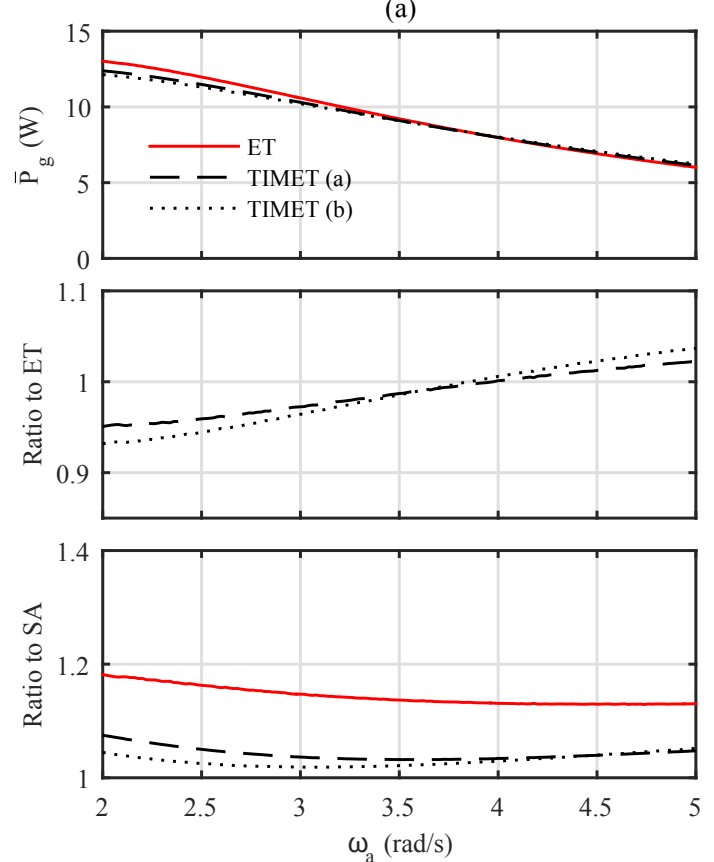
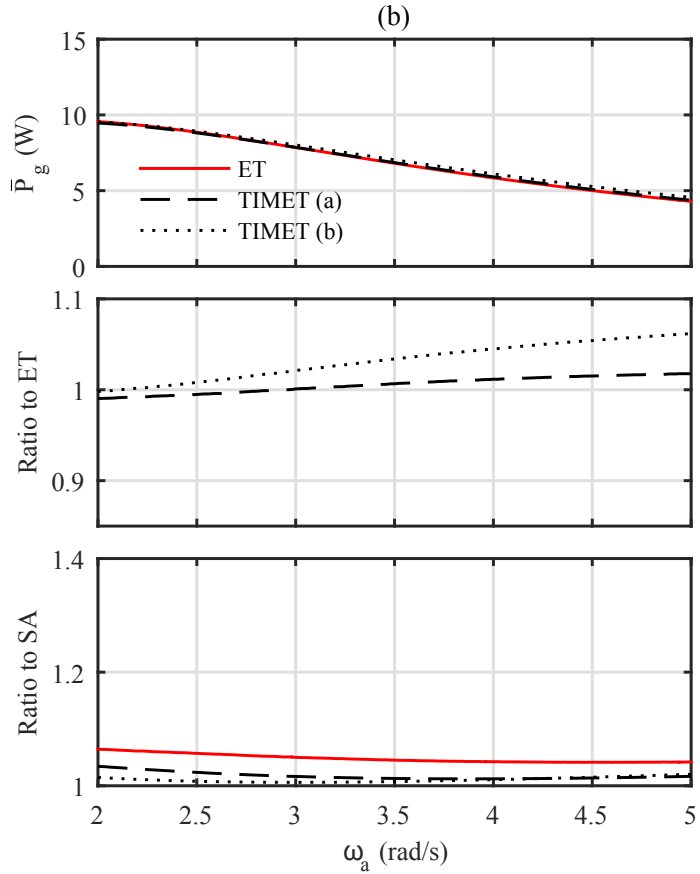

Figure 9: Comparisons of the energy harvesters obtained by the PG control for $\zeta_{a}=1$; (a) $R=5 \Omega$, (b) $R=20 \Omega$.

1430. arXiv:http://jim.sagepub.com/content/24/11/1405.full.pdf+html, doi:10.1177/1045389X13486707.

[7] L. Zuo, B. Scully, J. Shestani, Y. Zhou, Design and characterization of an electromagnetic energy harvester for vehicle suspensions, Smart Material Structures 19 (4) (2010) 045003. doi:10.1088/0964-1726/19/4/045003.

[8] C. Nagode, M. Ahmadian, S. Taheri, Effective energy harvesting devices for railroad applications, in: Proc. SPIE, Vol. 7643, 2010, pp. 76430X-76430X10. doi: $10.1117 / 12.847866$.

[9] S. M. Lattanzio, J. T. Scruggs, Maximum power generation of a wave energy converter in a stochastic environment, in: Control Applications (CCA), 2011 IEEE International Conference on, 2011, pp. 1125-1130. doi:10.1109/CCA.2011.6044428.

[10] X. Tang, L. Zuo, A. Kareem, Assessment of energy potential and vibration mitigation of regenerative tuned mass dampers on wind excited tall build- 

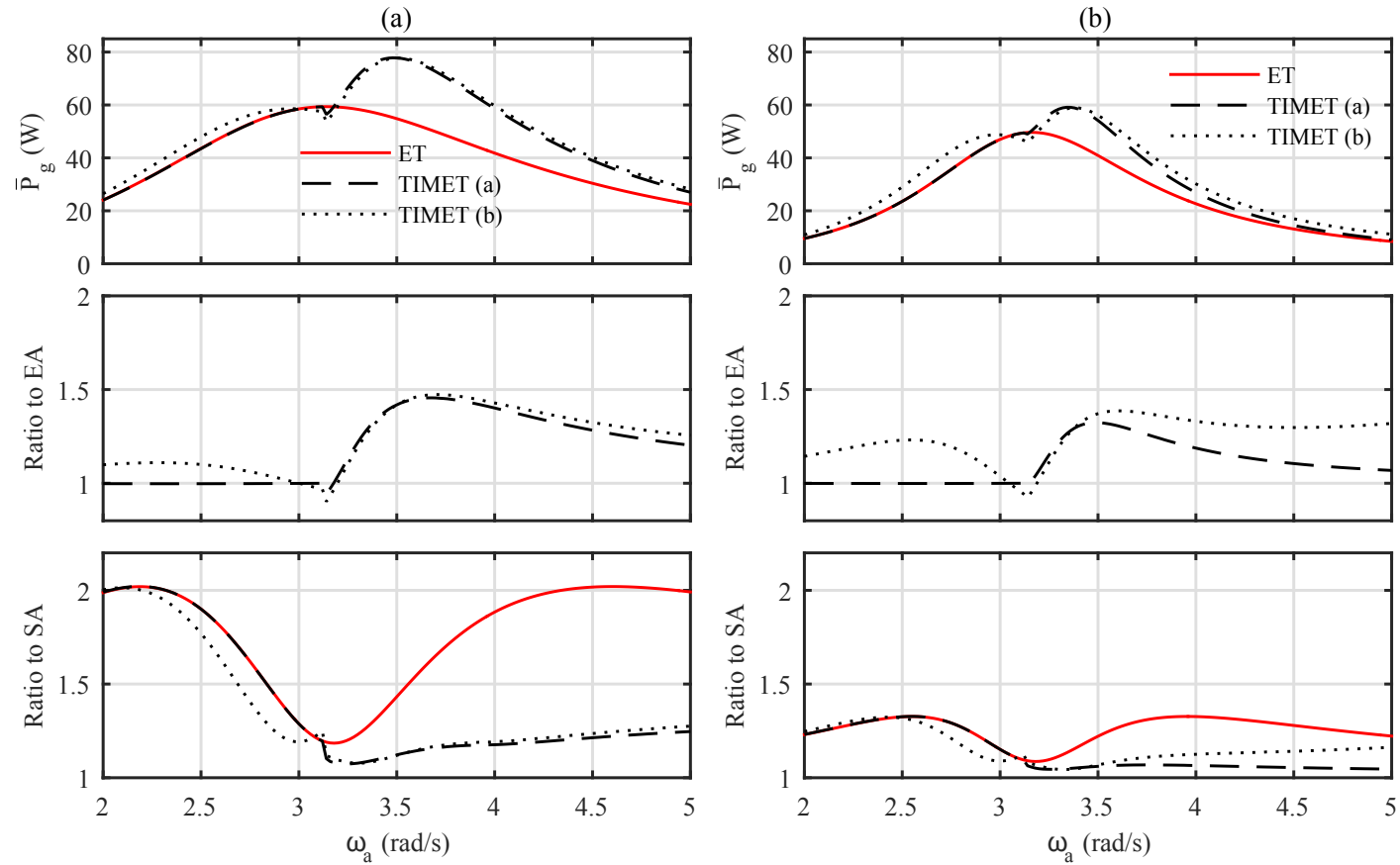

Figure 10: Comparisons of the energy harvesters obtained by the LQG control for for $\zeta_{a}=0.05$; (a) $R=5 \Omega$, (b) $R=20 \Omega$.

ings, in: the ASME 2011 International Design Engineering Technical Conferences and Computers and Information in Engineering Conference, no. 18, Washington, DC, 2011, pp. 333-342.

[11] K. Makihara, J. Onoda, T. Miyakawa, Low energy dissipation electric circuit for energy harvesting, Smart Materials and Structures 15 (5) (2006) 1493.

[12] J. K. Ward, S. Behrens, Adaptive learning algorithms for vibration energy harvesting, Smart Materials and Structures 17 (3) (2008) 035025.

[13] J. T. Scruggs, An optimal stochastic control theory for distributed energy harvesting networks, Journal of Sound and Vibration 320 (4 - 5) (2009) 707 - 725. doi:http://dx.doi.org/10.1016/j.jsv.2008.09.001.

[14] K. Ikago, K. Saito, N. Inoue, Seismic control of single-degree-of-freedom structure using tuned viscous mass damper, Earthquake Engineering \& Structural Dynamics 41 (3) (2012) 453-474. doi:10.1002/eqe.1138. 
(a)
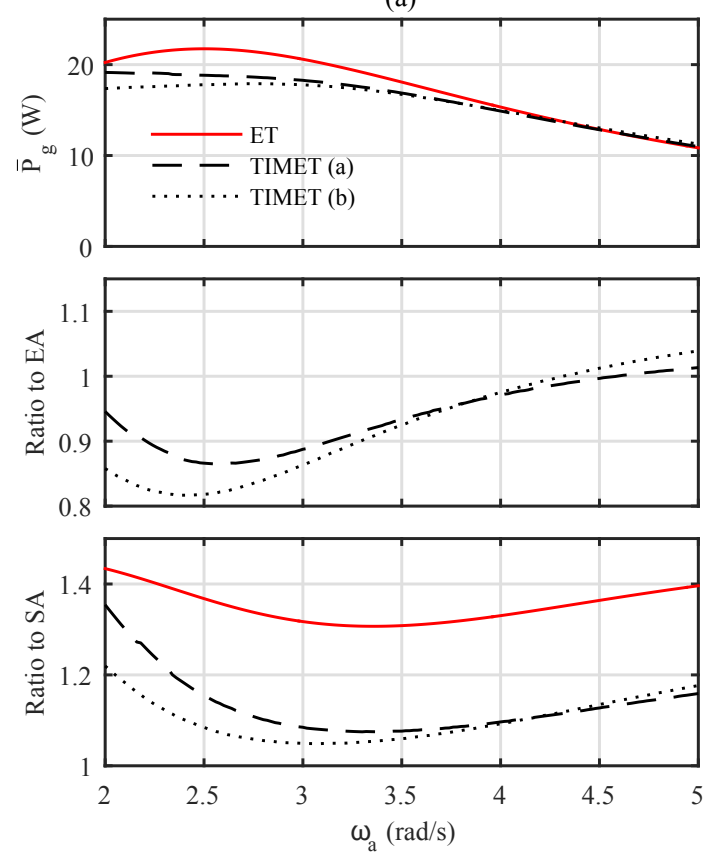

(b)
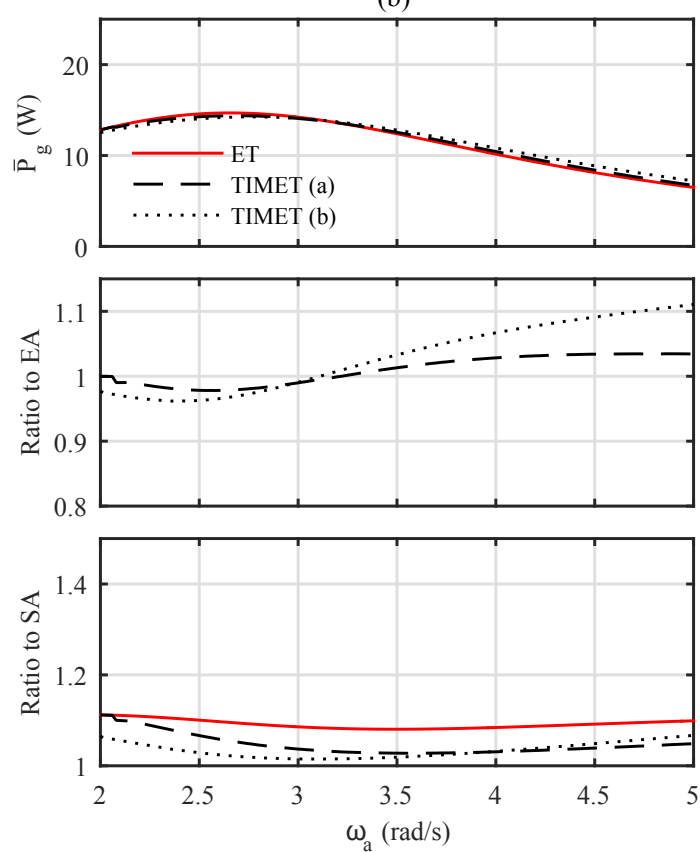

Figure 11: Comparisons of the energy harvesters obtained by the LQG control for for $\zeta_{a}=0.5$; (a) $R=5 \Omega$, (b) $R=20 \Omega$.

[15] T. Asai, K. Ikago, Y. Araki, Outrigger tuned viscous mass damping system for high-rise buildings subject to earthquake loadings, in: 6AESE/11ANCRiSST Joint Conference, 2015.

[16] M. C. Smith, Synthesis of mechanical networks: the inerter, IEEE Transactions on Automatic Control 47 (10) (2002) 1648-1662. doi:10.1109/TAC.2002.803532.

[17] I. F. Lazar, S. Neild, D. Wagg, Using an inerter-based device for structural vibration suppression, Earthquake Engineering \& Structural Dynamics 43 (8) (2014) 1129-1147. doi:10.1002/eqe.2390.

URL http://dx.doi.org/10.1002/eqe.2390

[18] A. Gonzalez-Buelga, L. R. Clare, S. A. Neild, J. Z. Jiang, D. J. Inman, An electromagnetic inerter-based vibration suppression device, Smart Materials and Structures 24 (5) (2015) 055015.

[19] I. Cassidy, J. Scruggs, S. Behrens, H. P. Gavin, Design and experimental 
(a)
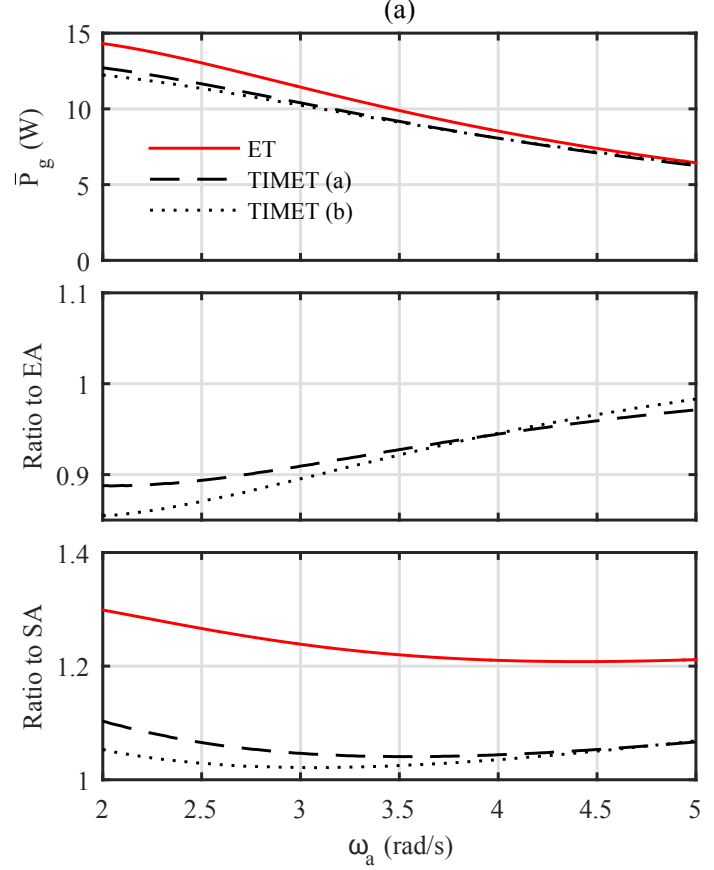
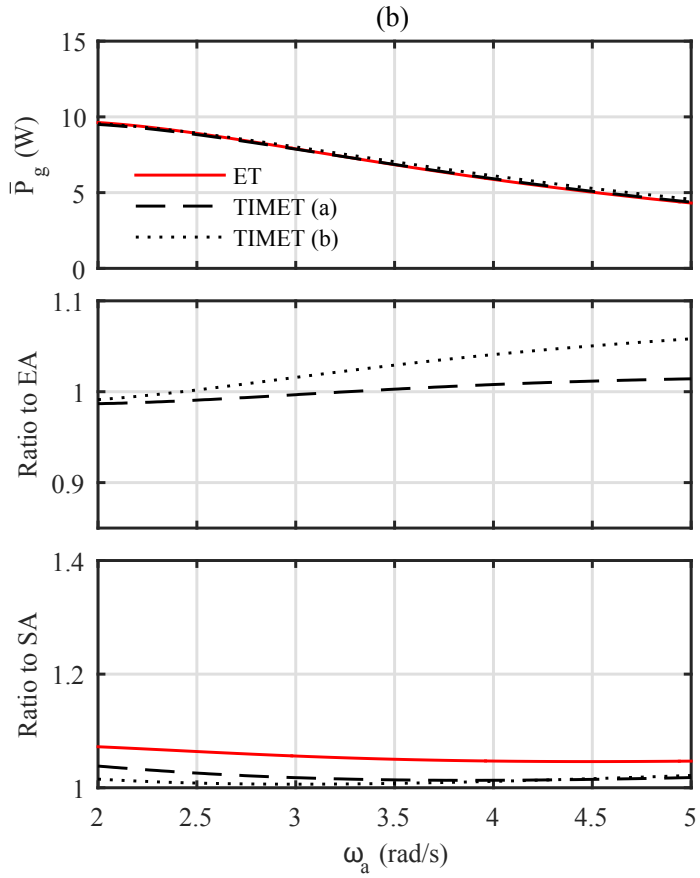

Figure 12: Comparisons of the energy harvesters obtained by the LQG control for $\zeta_{a}=1$; (a) $R=5 \Omega$, (b) $R=20 \Omega$.

characterization of an electromagnetic transducer for large-scale vibratory energy harvesting applications, Journal of Intelligent Material Systems and Structuresdoi:10.1177/1045389X11421824.

[20] I. L. Cassidy, J. T. Scruggs, Nonlinear stochastic controllers for power-flowconstrained vibratory energy harvesters, Journal of Sound and Vibration 332 (13) (2013) $3134-3147$.

[21] J. T. Scruggs., On the causal power generation limit for a vibratory energy harvester in broadband stochastic response, Journal of Intelligent Material Systems and Structures 21 (13) (2010) 12491262. arXiv:http://jim.sagepub.com/content/21/13/1249.full.pdf+html, doi:10.1177/1045389X10361794.

[22] R. Erickson, D. Maksimovic, Fundamentals of Power Electronics, Power electronics, Springer US, 2001.

[23] T. Paing, J. Shin, R. Zane, Z. Popovic, Resistor emulation approach to low- 
power rf energy harvesting, IEEE Transactions on Power Electronics 23 (3) (2008) 1494-1501. doi:10.1109/TPEL.2008.921167.

[24] N. Kong, D. S. Ha, A. Erturk, D. J. Inman, Resistive impedance matching circuit for piezoelectric energy harvesting, Journal of Intelligent Material Systems and Structures 21 (13) (2010) 12931302. arXiv:http://jim.sagepub.com/content/21/13/1293.full.pdf+html, doi:10.1177/1045389X09357971.

[25] P. Dorato, C. Abdallah, V. Cerone, Linear Quadratic Control: An Introduction, Krieger Publishing Company, 1995.

[26] R. Stengel, Optimal Control and Estimation, Dover books on advanced mathematics, Dover Publications, 1986.

[27] R. E. Skelton, T. Iwasaki, D. E. Grigoriadis, A unified algebraic approach to control design, CRC Press, 1997.

[28] T. Shimomura, T. Fujii, Multiobjective control design via successive overbounding of quadratic terms, in: Decision and Control, 2000. Proceedings of the 39th IEEE Conference on, Vol. 3, 2000, pp. 2763-2768. doi:10.1109/CDC.2000.914226.

[29] M. C. de Oliveira, J. F. Camino, R. E. Skelton, A convexifying algorithm for the design of structured linear controllers, in: Decision and Control, 2000. Proceedings of the 39th IEEE Conference on, Vol. 3, 2000, pp. 2781-2786. doi:10.1109/CDC.2000.914229.

[30] E. C. Warner, J. T. Scruggs, Control of vibratory networks with passive and regenerative systems, in: American Control Conference (ACC), 2015, 2015, pp. 5502-5508. doi:10.1109/ACC.2015.7172200. 